\title{
Rapid Transformation of Alkyl Halides into Symmetrical Disulfides Using Sodium Sulfide and Carbon Disulfide
}

\author{
Ishani Bhaumik \\ Anup Kumar Misra* \\ Division of Molecular Medicine, Bose Institute, P-1/12, C. I. T. \\ Scheme VII M, Kolkata 700054, India \\ akmisra69@gmail.com
}

\author{
RX $\underset{\text { DMF, r.t., 2-5 min }}{\stackrel{\mathrm{CS}_{2} / \mathrm{Na}_{2} \mathrm{~S} \cdot \mathrm{H}_{2} \mathrm{O}}{\longrightarrow}}$ RSSR \\ $86-96 \% 15$ compounds \\ fast \\ $\mathrm{R}=$ alkyl, glycosyl, glycosylalkyl \\ $\mathrm{X}=$ halide
}

\author{
Received: 06.06.2017 \\ Accepted after revision: 29.08.2017 \\ Published online: 14.09 .2017 \\ DOI: 10.1055/s-0036-1588573; Art ID: so-2017-d0025-I \\ License terms: (C) $९ \$$
}

Abstract An efficient one-pot reaction has been developed for the preparation of symmetrical disulfide derivatives directly from alkyl halides by reaction with a combination of sodium sulfide and carbon disulfide without requirement for any catalyst.

Key words alkyl halides, carbohydrates, disulfides, sodium sulfide, carbon disulfide

Organic disulfides are an important class of compounds that have applications in organic synthesis, ${ }^{1}$ biological studies, ${ }^{2}$ drug delivery, ${ }^{3}$ and the polymer industry. ${ }^{4}$ Stabilization of protein structure due to the formation of disulfide bridges is common in biological systems ${ }^{5}$ and ligation through the formation of disulfide linkage is often used in the functionalization of proteins. ${ }^{6}$ Several bioactive molecules contain disulfide bonds as active pharmacophores. ${ }^{7}$ Disulfide compounds are used as vulcanizing agents for rubber. ${ }^{8}$

Several reports have appeared on the preparation of disulfides by the oxidation of thiols using a variety of reagents, ${ }^{9}$ electrochemical oxidation ${ }^{10}$ and enzymatic reaction. ${ }^{11}$ They have also been prepared from thiol acetates using clayfen under solvent-free conditions. ${ }^{12}$ Alkyl halides could be considered as lower odor alternatives of thiols for the preparation of disulfide derivatives. The preparation of disulfides starting from alkyl halides using sodium sulfide in the presence of a phase-transfer catalyst ${ }^{13}$ and hexachloroethane, carbon tetrachloride or thiourea in PEG medium has been reported. ${ }^{14}$ In addition, disulfides have been prepared from alcohols, ${ }^{15}$ thiocyanates, ${ }^{16}$ epoxides, ${ }^{17}$ aziri- dines, ${ }^{18}$ and $S$-alkylthiosulfates (Bunte salts). ${ }^{19}$ Despite their synthetic utilities, the above mentioned approaches suffer from shortcomings, which include the use of malodorous thiols, requirement of special reaction conditions, hazardous reagents, extended reaction times, high temperatures, unsatisfactory yield and limited substrate scope. In the search for efficient reaction conditions for the preparation of symmetrical disulfides, we have explored the reaction of alkyl halides with a combination of sodium sulfide $\left(\mathrm{Na}_{2} \mathrm{~S} \cdot 9 \mathrm{H}_{2} \mathrm{O}\right)$ and carbon disulfide $\left(\mathrm{CS}_{2}\right)$ (Scheme 1). Recently, we have used the combination of $\mathrm{Na}_{2} \mathrm{~S} \cdot 9 \mathrm{H}_{2} \mathrm{O}$ and $\mathrm{CS}_{2}$ as a surrogate of hydrogen sulfide for the formation of glycosyl thiol derivatives. ${ }^{20}$ During the preparation of glycosyl thiols it was observed that variation of the ratio of $\mathrm{Na}_{2} \mathrm{~S} \cdot 9 \mathrm{H}_{2} \mathrm{O}$ and $\mathrm{CS}_{2}$ as well as the presence of substituents on the sugar ring led to the formation of disulfide derivatives. In fact, sodium sulfide has been used to react with alkyl halides to produce symmetrical sulfides in the presence of a phase-transfer catalyst. ${ }^{13}$ To our satisfaction, we found that the disulfide derivatives were formed almost instantly by mixing the substrates and the reagent system without formation of symmetrical thioethers as by-products. In this communication, we present the fast, efficient preparation of symmetrical disulfide derivatives directly from alkyl halides in excellent yield.

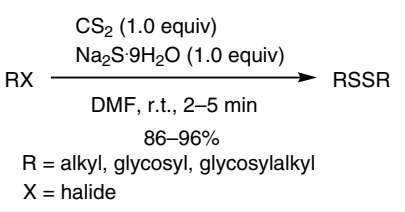

Scheme 1 Synthesis of symmetrical disulfides from alkyl halides using a combination of sodium sulfide and carbon disulfide at room temperature 
In initial experiments, benzyl bromide was added to a varied stoichiometric combination $\mathrm{Na}_{2} \mathrm{~S} \cdot 9 \mathrm{H}_{2} \mathrm{O}$ and $\mathrm{CS}_{2}$ in DMF at room temperature. It was observed that treatment of benzyl bromide $(1.0 \mathrm{mmol})$ with a combination of $\mathrm{Na}_{2} \mathrm{~S} \cdot 9 \mathrm{H}_{2} \mathrm{O}(1.0 \mathrm{mmol})$ and $\mathrm{CS}_{2}(1.0 \mathrm{mmol})$ in DMF at room temperature instantaneously furnished dibenzyl disulfide 7 in $96 \%$ yield. Reduction of the quantity of either $\mathrm{Na}_{2} \mathrm{~S} \cdot 9 \mathrm{H}_{2} \mathrm{O}$ or $\mathrm{CS}_{2}$ resulted in the formation of product $\mathbf{7}$ in poor yield due to the formation of thioether derivatives. However, increasing the quantity of the reagents did not improve the yield significantly. The reaction did not take place in the absence of either $\mathrm{Na}_{2} \mathrm{~S} \cdot 9 \mathrm{H}_{2} \mathrm{O}$ or $\mathrm{CS}_{2}$ (Table 1). Notably, the reaction does not require any metallic or phase-transfer catalyst. Commonly used solvents such as $\mathrm{CH}_{2} \mathrm{Cl}_{2}, \mathrm{THF}, \mathrm{CH}_{3} \mathrm{CN}$, DMF, DMSO, $\mathrm{CH}_{3} \mathrm{OH}$, and $\mathrm{H}_{2} \mathrm{O}$ were screened for their suitability to carry out the reaction. Excellent yields of $\mathbf{7}$ were obtained by carrying out the reaction in DMF and DMSO due to the high solubility of the reagents compared with other solvents (Table 1). However, DMF was considered as the preferred solvent due to the drawbacks associated with DMSO such as high boiling point, unpleasant odor and scope for formation of by-products. Although earlier $\mathrm{Na}_{2} \mathrm{~S} \cdot 9 \mathrm{H}_{2} \mathrm{O}$-mediated thiolation reactions were carried out in water or $\mathrm{CH}_{3} \mathrm{OH}$ at high temperature or in the presence of a phase-transfer catalyst, under these conditions a satisfactory yield of the product was not obtained; presumably due to the loss of carbon disulfide at high temperature. Following the optimization studies, a series of symmetrical disulfide derivatives was prepared in excellent yield (Table 2). ${ }^{23}$ The reaction conditions were also successfully applied for the preparation of the 0 -glycosylated alkyl disulfide derivatives. The functional groups present in the sugar moieties were compatible with the reaction conditions. A variety of alkyl halides were used for the preparation of disulfide de-

Table 1 Reaction of Benzyl Bromide with $\mathrm{Na}_{2} \mathrm{~S} \cdot 9 \mathrm{H}_{2} \mathrm{O}$ and $\mathrm{CS}_{2}$ in Different Solvents at Room Temperature

\begin{tabular}{lccccc}
\hline Entry & $\mathrm{Na}_{2} \mathrm{~S} \cdot 9 \mathrm{H}_{2} \mathrm{O}$ (equiv) & $\mathrm{CS}_{2}$ (equiv) & Solvent & Time (min) & Yield (\%) \\
\hline $\mathbf{1}$ & $\mathbf{1 . 0}$ & $\mathbf{1 . 0}$ & DMF & $\mathbf{2}$ & $\mathbf{9 6}$ \\
2 & 1.0 & 0.5 & $\mathrm{DMF}$ & 10 & 55 \\
3 & 0.5 & 1.0 & $\mathrm{DMF}$ & 10 & 65 \\
4 & 1.5 & 1.5 & $\mathrm{DMF}$ & 2 & 96 \\
5 & 1.0 & 1.0 & $\mathrm{DMSO}$ & 2 & 95 \\
6 & 1.0 & 1.0 & $\mathrm{THF}$ & 30 & 68 \\
7 & 1.0 & 1.0 & $\mathrm{CH}_{3} \mathrm{CN}$ & 60 & 70 \\
8 & 1.0 & 1.0 & $\mathrm{CH}_{2} \mathrm{Cl}_{2}$ & 120 & 48 \\
9 & 1.0 & 1.0 & $\mathrm{CH}_{3} \mathrm{OH}$ & 10 & 64 \\
10 & 1.0 & 1.0 & $\mathrm{H}_{2} \mathrm{O}$ & 720 & 52 \\
11 & - & 1.0 & $\mathrm{DMF}^{2}$ & 720 & - \\
12 & 1.0 & - & $\mathrm{DMF}^{2}$ & 720 & 20 \\
\hline
\end{tabular}

rivatives. The reaction is exceptionally fast and disulfide derivatives were obtained exclusively within 2-5 min. The reaction has been successfully applied for a scaled-up (20 g) preparation of dibenzyl disulfide (7) in excellent yield (Table 2). All products were unambiguously characterized by spectroscopic analysis. ${ }^{24}$

A plausible mechanistic pathway is presented in Scheme 2. Presumably, the reaction of $\mathrm{Na}_{2} \mathrm{~S} \cdot 9 \mathrm{H}_{2} \mathrm{O}$ and $\mathrm{CS}_{2}$ generates a carbonotrithioate ion in situ, which displaces the halide ion in the alkyl halide by nucleophilic substitution to furnish an alkyl thiolate ion after regenerating $\mathrm{CS}_{2}$. Finally, oxidative condensation of alkyl thiolates results in the formation of the symmetrical disulfide.

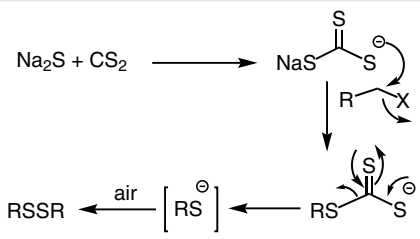

Scheme 2 Plausible mechanism for the formation of symmetrical disulfides

In summary, an exceptionally fast reaction has been developed for the direct preparation of symmetrical disulfide derivatives in excellent yield from alkyl and glycosylalkyl halides by using a combination of $\mathrm{Na}_{2} \mathrm{~S} \cdot 9 \mathrm{H}_{2} \mathrm{O}$ and $\mathrm{CS}_{2} \cdot{ }^{23}$ This clean, catalyst-free reaction is suitable for scale-up. By applying these reaction conditions, a diverse range of disulfide derivatives of non-commercial thiols can also be prepared in excellent yield.

\section{Acknowledgment}

I.B. thanks CSIR, New Delhi for providing a Senior Research Fellowship. This work was supported by SERB, New Delhi (Project No. EMR/2015/000282).

\section{References}

(1) (a) Karchmer, J. H. The Analytical Chemistry of Sulfur and Its Compounds; Wiley: New York, 1972. (b) Oae, S. Organic Sulfur Chemistry: Structure and Mechanism; CRC Press: Boca Raton, FL, 1991. (c) Johnson, J. R.; Bruce, W. F.; Dutcher, J. D. J. Am. Chem. Soc. 1943, 65, 2005.

(2) (a) Bodanszky, M. Principles of Peptide Synthesis; Springer: Berlin, 1984, Chap. 4, 119-157. (b) Patai, S. Chemistry of the Thiol Groups; Wiley \& Sons: New York, 1974, 785.

(3) (a) Saito, G.; Swanson, J. A.; Lee, K. D. Adv. Drug Delivery Rev. 2003, 55, 199. (b) Lee, M. H.; Sessler, J. L.; Kim, J. S. Acc. Chem. Res. 2015, 48, 2935

(4) (a) Graf, T. A.; Yoo, J.; Brummett, A. B.; Lin, R.; Wohlgenannt, M.; Quinn, D.; Bowden, N. B. Macromolecules 2012, 45, 8193. (b) Gyarmati, B.; Némethy, Á.; Szilágui, Eur. Polym. J. 2013, 49, 1268. (c) Zelikin, A. N.; Quinn, J. F.; Caruso, F. Biomacromolecules 2006, 7, 27. 
Table 2 Preparation of Disulfides from Alkyl Halides using $\mathrm{Na}_{2} \mathrm{~S} \cdot 9 \mathrm{H}_{2} \mathrm{O}(1.0 \mathrm{mmol})$ and $\mathrm{CS}_{2}(1.0 \mathrm{mmol})$ in DMF at Room Temperature

$$
\mathrm{RX}+\mathrm{CS}_{2}+\mathrm{Na}_{2} \mathrm{~S} \cdot 9 \mathrm{H}_{2} \mathrm{O} \underset{\text { r.t. }}{\stackrel{\mathrm{DMF}}{\longrightarrow}} \mathrm{RSSR}
$$

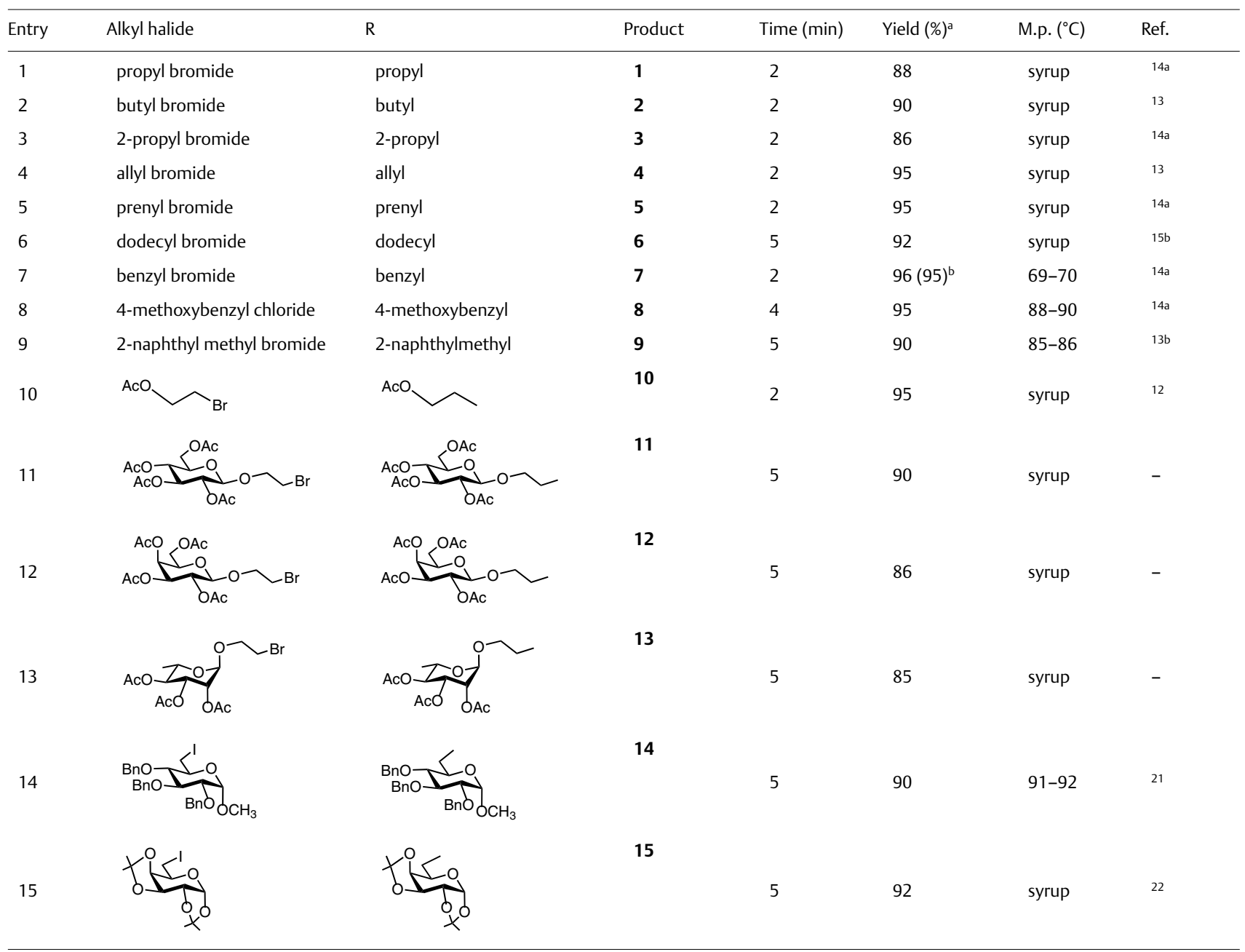

\footnotetext{
a Isolated yield.
}

b Scale up preparation.

(5) (a) Trivedi, M. V.; Laurence, J. S.; Siahaan, T. J. Curr. Protein Pept. Sci. 2009, 10, 614. (b) Oka, O. B. V.; Bulleid, N. J. Biochim. Biophys. Acta, Mol. Cell Res. 2013, 1833, 2425.

(6) (a) Marshall, C. J.; Agarwal, N.; Kalia, J.; Grosskopf, V. A.; McGrath, N. A.; Abbott, N. L.; Raines, R. T.; Shusta, E. V. Bioconjugate Chem. 2013, 24, 1634. (b) van Vught, R.; Pieters, R. J.; Breukink, E. Comput. Struct. Biotechnol. J. 2014, 9, e201402001.

(7) (a) Góngora-benitez, M.; Tulla-Puche, J.; Albericio, F. Chem. Rev. 2014, 114, 901. (b) Brady, R. M.; Baell, J. B.; Norton, R. S. Mar. Drugs 2013, 11, 2293.

(8) (a) Adhikari, B.; De, D.; Maiti, S. Prog. Polym. Sci. 2000, 25, 909. (b) Sonavane, S. U.; Chidambaram, M.; Almog, J.; Sasson, Y. Tetrahedron Lett. 2007, 48, 6048.

(9) (a) Iranpoor, N.; Firouzabadi, H.; Pourali, A. R. Tetrahedron 2002, 58, 5179. (b) Silveira, C. C.; Mendes, S. R. Tetrahedron Lett. 2007, 48, 7469. (c) Akdag, A.; Webb, T.; Worley, S. D. Tetrahedron Lett.
2006, 47, 3509. (d) Olah, G. A.; Arvanaghi, M.; Vankar, Y. D. Synthesis 1979, 721. (e) Mckillop, A.; Koyuncu, D.; Krief, A.; Dumont, W.; Renier, P.; Trabelsi, M. Tetrahedron Lett. 1990, 31, 5007. (f) Fujihara, H.; Mima, H.; Ikemori, M.; Furukawa, N. J. Am. Chem. Soc. 1991, 113, 6337. (g) Kirihara, M.; Okubo, K.; Uchiyama, T.; Kato, Y.; Ochiai, Y.; Matsushita, S.; Hatano, A.; Kanamori, K. Chem. Pharm. Bull. 2004, 52, 625. (h) Ali, M. H.; McDermott, M. Tetrahedron Lett. 2002, 43, 6271. (i) Iranpoor, N.; Zeynizadeh, B. Synthesis 1999, 49. (j) Sato, T.; Otera, J.; Nozaki, H. Tetrahedron Lett. 1990, 31, 3591. (k) Misra, A. K.; Agnihotri, G. Synth. Commun. 2004, 34, 1079. (l) Kirihara, M.; Asai, Y.; Ogawa, S.; Noguchi, T.; Hatano, A.; Hirai, Y. Synthesis 2007, 3286. (m) Hosseinpoor, F.; Golchoubian, H. Catal. Lett. 2006, 111, 165. (n) Lenardao, E. J.; Lara, R. G.; Silva, M. S.; Raquel, G.; Jacob, R. G.; Perin, G. Tetrahedron Lett. 2007, 48, 7668. (o) Firouzabadi, H.; Mottghinejad, E.; Seddighi, M. Synthesis 1989, 378. 
(10) Sergio, L. S.; Pardini, V. L.; Viertler, H. Synth. Commun. 1990, 20 , 393.

(11) Rao, K. R.; Kumar, H. M. S. Bioorg. Med. Chem. Lett. 1991, 1, 507.

(12) Meshram, H. M. Tetrahedron Lett. 1993, 34, 2521.

(13) (a) Sonavane, S. U.; Chidambaram, M.; Almog, J.; Sasson, Y. Tetrahedron Lett. 2007, 48, 6048. (b) Wang, J.-X.; Cui, W.; Hu, Y. Synth. Commun. 1995, 25, 3573.

(14) (a) Abbasi, M.; Mohammadizadeh, M. R.; Moosavi, H.; Saeedi, N. Synlett 2015, 26, 1185. (b) Firouzabadi, H.; Iranpoor, N.; Abbasi, M. Tetrahedron Lett. 2010, 51, 508.

(15) (a) Sinha, S.; Ilankumaran, P.; Chandrasekaran, S. Tetrahedron 1999, 55, 14769. (b) Iranpoor, N.; Firouzabadi, H.; Khalili, D. Tetrahedron Lett. 2012, 53, 6913.

(16) Prabhu, K. R.; Ramesha, A. R.; Chandrasekaran, S. J. Org. Chem. 1995, 60, 7142 .

(17) Devan, N.; Sridhar, P. R.; Prabhu, K. R.; Chandrasekaran, S. J. Org. Chem. 2002, 67, 9417.

(18) Sureshkumar, D.; Gunasundari, T.; Ganesh, V.; Chandrasekaran, S. J. Org. Chem. 2006, 72, 2106.

(19) (a) Liu, Y.; Zheng, H.; Xu, D.; Xu, Z.; Zhang, Y. Synlett 2006, 2492. (b) Wang, L.; Zhang, Y. Tetrahedron 1999, 55, 10695.

(20) Jana, M.; Misra, A. K. J. Org. Chem. 2013, 78, 2680.

(21) Liu, C.-Y.; Chen, H.-L.; Ko, C.-M.; Chen, C.-T. Tetrahedron 2011, 67, 872.

(22) Adinolfi, M.; Capasso, D.; Gaetano, S. D.; Iadonisi, A.; Leone, L.; Pastore, A. Org. Biomol. Chem. 2011, 9, 6278.

(23) Typical experimental procedure for the preparation of symmetrical dialkyl disulfides: To a solution of $\mathrm{Na}_{2} \mathrm{~S} \cdot 9 \mathrm{H}_{2} \mathrm{O}(1.0$ $\mathrm{mmol})$ in DMF $(2 \mathrm{~mL})$ was added $\mathrm{CS}_{2}(1.0 \mathrm{mmol})$ at room temperature. The alkyl halide $(1.0 \mathrm{mmol})$ was added to the dark-red reaction mixture at room temperature with vigorous stirring. The color of the reaction mixture changed from red to yellow. The reaction mixture was stirred for the appropriate time (Table $2)$, then poured into water and extracted with $\mathrm{Et}_{2} \mathrm{O}(2 \times 25 \mathrm{~mL})$.
The combined organic layers were dried over $\mathrm{Na}_{2} \mathrm{SO}_{4}$, filtered and concentrated under reduced pressure. The crude product was purified over $\mathrm{SiO}_{2}$ using hexane-EtOAc $(15: 1)$ as eluant to give the pure dialkyl disulfide derivative (Table 2)

(24) Spectroscopic data of novel products:

\section{Di[2-0-(2,3,4,6-tetra-O-acetyl- $\beta$-D-glucopyranosyl)ethyl]}

Disulfide (11): Yield: $733 \mathrm{mg}$ (90\%); Colorless oil; ${ }^{1} \mathrm{H}$ NMR (500 $\left.\mathrm{MHz}, \mathrm{CDCl}_{3}\right): \delta=5.18(\mathrm{t}, J=7.5 \mathrm{~Hz}, 2 \mathrm{H}), 5.08(\mathrm{t}, J=9.5 \mathrm{~Hz}, 2 \mathrm{H})$, $4.97(\mathrm{~m}, 2 \mathrm{H}), 4.55(\mathrm{~d}, J=9.0 \mathrm{~Hz}, 2 \mathrm{H}), 4.26(\mathrm{dd}, J=4.5,8.0 \mathrm{~Hz}$, $2 \mathrm{H}), 4.14-3.95(\mathrm{~m}, 4 \mathrm{H}), 3.89-3.60$ ( $\mathrm{m}, 4 \mathrm{H}), 2.96-2.71(\mathrm{~m}, 4 \mathrm{H})$, 2.09, 2.06, 2.02, $2.00(4 \times \mathrm{s}, 24 \mathrm{H}) ;{ }^{13} \mathrm{C}$ NMR $\left(125 \mathrm{MHz}, \mathrm{CDCl}_{3}\right)$ : $\delta=170.2$ (2 C), 169.9 (2 C), 169.0 (2 C), 168.9 (2 C), 100.7 (2 C), 72.7 (2 C), 72.6 (2 C), 71.8 (2 C), 69.6 (4 C), 67.6 (2 C), 61.7 (2 C), 38.3 (2 C), 20.5 (8 C); HRMS (ESI): $m / z[M+N a]^{+}$calcd. for $\mathrm{C}_{32} \mathrm{H}_{46} \mathrm{O}_{20} \mathrm{~S}_{2}$ : 837.1922; found: 837.1916.

Di[2-O-(2,3,4,6-tetra-O-acetyl- $\beta$-D-galactopyranosyl)ethyl] Disulfide (12): Yield: $700 \mathrm{mg}(86 \%)$; Colorless oil; ${ }^{1} \mathrm{H}$ NMR (500 $\left.\mathrm{MHz}, \mathrm{CDCl}_{3}\right): \delta=5.39-5.31(\mathrm{~m}, 2 \mathrm{H}), 5.20-5.13(\mathrm{~m}, 2 \mathrm{H}), 5.05-$ $4.95(\mathrm{~m}, 2 \mathrm{H}), 4.50(\mathrm{~d}, J=8.0 \mathrm{~Hz}, 2 \mathrm{H}), 4.19-4.09(\mathrm{~m}, 4 \mathrm{H}), 4.05-$ $3.89(\mathrm{~m}, 2 \mathrm{H}), 3.81-3.61(\mathrm{~m}, 2 \mathrm{H}), 2.91-2.71(\mathrm{~m}, 4 \mathrm{H}), 2.16,2.07$, 2.05, $1.98(4 \times \mathrm{s}, 24 \mathrm{H}) ;{ }^{13} \mathrm{C}$ NMR $\left(125 \mathrm{MHz}, \mathrm{CDCl}_{3}\right): \delta=170.1(2$ C), 170.0 (2 C), 169.9 (2 C), 169.2 (2 C), 101.4 (2 C), 70.8 (4 C), 70.7 (2 C), 68.7 (4 C), 66.9 (2 C), 61.1 (2 C), 20.7 (8 C); HRMS (ESI): $\mathrm{m} / \mathrm{z}[\mathrm{M}+\mathrm{Na}]^{+}$calcd. for $\mathrm{C}_{32} \mathrm{H}_{46} \mathrm{O}_{20} \mathrm{~S}_{2}: 837.1922$; found: 837.1917.

Di-[2-0-(2,3,4-tri-0-acetyl- $\alpha$-L-rhamnopyranosyl)ethyl] Disulfide (13): Yield: $594 \mathrm{mg}$ (85\%); Colorless oil; ${ }^{1} \mathrm{H}$ NMR (500 $\left.\mathrm{MHz}, \mathrm{CDCl}_{3}\right): \delta=5.31-5.19(\mathrm{~m}, 4 \mathrm{H}), 5.05(\mathrm{t}, J=9.5 \mathrm{~Hz}, 2 \mathrm{H}), 4.76$ (s, $2 \mathrm{H}), 3.98-3.88(\mathrm{~m}, 2 \mathrm{H}), 3.85-3.69(\mathrm{~m}, 4 \mathrm{H}), 2.98-2.81(\mathrm{~m}$, $4 \mathrm{H}), 2.15,2.08,1.98(3 \times \mathrm{s}, 18 \mathrm{H}), 1.22(\mathrm{~d}, J=6.0 \mathrm{~Hz}, 6 \mathrm{H})$; ${ }^{13} \mathrm{C} \mathrm{NMR}\left(125 \mathrm{MHz}, \mathrm{CDCl}_{3}\right): \delta=169.9(2 \mathrm{C}), 169.8(2 \mathrm{C}), 169.7(2$ C), 97.5 (2 C), 70.9 (2 C), 70.6 (2 C), 69.7 (2 C), 69.0 (2 C), 66.6 (2 C), 66.3 (2 C), 38.2 (2 C), 20.8 (6 C), 17.4 (2 C); HRMS (ESI): $\mathrm{m} / \mathrm{z}[\mathrm{M}+\mathrm{Na}]^{+}$calcd. for $\mathrm{C}_{28} \mathrm{H}_{42} \mathrm{O}_{16} \mathrm{~S}_{2}: 721.1812$; found: 721.1806 